\title{
A Distributed Joint Channel-Assignment, Scheduling and Routing Algorithm for Multi-Channel Ad Hoc Wireless Networks
}

\author{
Xiaojun Lin and Shahzada Rasool \\ School of Electrical and Computer Engineering, Purdue University \\ West Lafayette, IN 47907, U.S.A. \\ \{linx,srasool\}@ecn.purdue.edu
}

\begin{abstract}
The capacity of ad hoc wireless networks can be substantially increased by equipping each network node with multiple radio interfaces that can operate on multiple non-overlapping channels. However, new scheduling, channelassignment, and routing algorithms are required to fully utilize the increased bandwidth in multi-channel multi-radio ad hoc networks. In this paper, we develop a fully distributed algorithm that jointly solves the channel-assignment, scheduling and routing problem. Our algorithm is an online algorithm, i.e., it does not require prior information on the offered load to the network, and can adapt automatically to the changes in the network topology and offered load. We show that our algorithm is provably efficient. That is, even compared with the optimal centralized and offline algorithm, our proposed distributed algorithm can achieve a provable fraction of the maximum system capacity. Further, the achievable fraction that we can guarantee is larger than that of some other comparable algorithms in the literature.
\end{abstract}

\section{INTRODUCTION}

Multi-channel multi-radio ad hoc wireless networks have recently received a substantial amount of interest, especially under the context of wireless mesh networks [1]-[13]. It has been shown that one can significantly increase the capacity of ad hoc wireless networks by equipping each network node with multiple radio interfaces that operate on multiple non-overlapping channels. This is motivated by some current wireless LAN standards (in particular, IEEE 802.11) where the entire frequency band is divided into multiple channels, and each radio can only access one channel at a time. Hence, if each network node has multiple radio interfaces, it can then utilize a larger amount of radio bandwidth, and hence achieves higher system capacity. Even if each node still has only one radio interface, by operating neighboring nodes at different channels, the amount of interference is reduced, which also leads to higher system capacity.

Such multi-channel multi-radio networks pose an interesting set of resource allocation problems, including (i) channelassignment: what are the set of channels that each node/link should operate on? (ii) scheduling: when should each link be activated at each channel? and (iii) routing: how to select paths that minimize interference and increase throughput? These three problems are inter-related with each other, and thus form a challenging cross-layer control problem across the MAC layer and the network layer.

In this work, we are interested in control protocols for multi-channel multi-radio ad hoc networks that achieve high system capacity. Although such control protocols for channelassignment, scheduling and routing can be obtained via the throughput-optimal algorithms in [14] and [15] that are known to achieve the maximum system capacity, these algorithms are centralized and often with exponential computationalcomplexity. Hence, they are not easy to implement in real systems. In this paper, we develop distributed and provablyefficient solutions to the above cross-layer control problem. By provably-efficient, we mean that for any offered load that a given multi-channel network can ever support (possibly by using the centralized and complex throughput-optimal algorithms of [14], [15]), our algorithm can guarantee to support at least a constant fraction of this offered load on the same network. In other words, our algorithm can achieve a provable fraction of the maximum system capacity. Our algorithm is an online algorithm, i.e., it does not require prior knowledge of the offered load, and can automatically track the changes in the network topology and offered load. The distributive and online nature of our solution, combined with its provable efficiency, differentiates our work from existing control algorithms in the literature for multi-channel multi-radio ad hoc networks that either do not guarantee provable performance bounds [1], [2], [5], [9]-[13]. or require centralized and offline solutions [7], [8], [10], [14], or only provide order-optimal scaling laws [4].

Our solution is perhaps most comparable to the polynomial complexity (but centralized) algorithm in [7], which is also shown to guarantee a certain fraction of the maximum system capacity. Compared with the centralized solution of [7], our control algorithm is not only distributed and much simpler, but also guarantees a higher fraction of the maximum system capacity (see the comparison at the end of Section IV-B for details). One of the key differences between our approach and that of [7] is our assumption that, if the number of radio interfaces of a network node is less than the number of channels, the network node can switch radios from one channel to another dynamically [1], [2], [5], [6], [8], [13]. In contrast, the work in [7] requires radio-channel assignment to be fixed. 
It does incur additional overheads in order to enable dynamic channel switching, which include both the hardware switching delay [1], and the protocol overheads for changing channelassignment. However, these overheads could be reduced by improved hardware technology and refined protocols. More importantly, we believe that our results provide a strong motivation to pursue such improved channel-switching hardwares and protocols because, by allowing dynamic channel switching, one can obtain control protocols (like the one developed in this paper) that are both simpler and with higher provable performance.

Our work is related to the recent progress in developing distributed and provably-efficient scheduling algorithms for single-channel multi-hop wireless networks [16]-[22]. However, as we will show in Section III-B, straight-forward extensions of these single-channel distributed scheduling algorithms to multi-channel networks may lead to very poor performance. The reason is that, in multi-channel networks, there may exist channel-diversity. That is, due to both frequencyselective multi-path fading and different amount of background interference, each link can have different rate at each channel. In Section III-B, we will provide examples to show that straight-forward extensions of a well-studied single-channel distributed scheduling algorithm, i.e., the Maximal Scheduling algorithm, can perform very poorly in multi-channel systems with channel diversity. In contrast, the algorithm that we develop in this paper can guarantee provable efficiency even with channel diversity.

Our work is also related to the opportunistic scheduling algorithms in cellular networks that use OFDM (Orthogonal Frequency Division Multiplexing). Note that in OFDM, the transmitter sends information over a large number of subcarriers (e.g., 52 sub-carriers in IEEE 802.11a). Hence, OFDM systems can also be viewed as a special type of multi-channel multi-radio wireless systems. Opportunistic scheduling algorithms can significantly improve the capacity of such systems by exploiting sub-carrier frequency-diversity (and also timediversity) [23]-[25]. However, such opportunistic scheduling algorithms for cellular networks cannot be extended directly to ad hoc wireless networks because of the distributive nature of ad hoc networks. On the other hand, the algorithm that we develop in this paper can be viewed as a distributed opportunistic scheduling algorithm for OFDM-based ad hoc wireless networks.

The rest of the paper is organized as follows. We first outline the network model in Section II. In Section III, we illustrate the important effect of channel diversity, and show that straightforward extensions of a single-channel distributed scheduling algorithm can perform very poorly in multi-channel networks with channel diversity. We then present our new distributed and provably-efficient algorithm in Section IV. Simulation results are presented in Section V. Then we conclude.

\section{SYSTEM MODEL}

Consider a wireless network with $N$ nodes and $L$ links. Each link corresponds to a pair of transmitter node and receiver node. Let $b(l)$ and $e(l)$ denote the transmitter node and the receiver node, respectively, of link $l$. Let $E(i)$ denote the set of all links originating or terminating at node $i$. There are $C$ frequency channels in the system. In order to take into account possible channel diversity, we use $r_{l}^{c}$ to denote the rate at which link $l$ can transfer data on channel $c$, provided that there are no interfering links transmitting on channel $c$ at the same time. The interference relationship is defined as follows. For each link $l$, there is a set $I_{l}$ of links that interfere with $l$. That is, if link $l$ and another link in $I_{l}$ are transmitting on the same channel at the same time, neither of the links can transfer any useful data. We assume that the interference relationship is symmetrical, i.e., $k \in I_{l}$ if and only if $l \in I_{k}$ for any two links $k$ and $l$. For simplicity, we adopt the convention that $l \in I_{l}$. Note that the interference relationship is identical over all channels. Further, the channels are non-overlapping. Hence, it is perfectly fine that link $l$ and another link $k \in I_{l}$ transmit at different channels at the same time. The above interference model is very general and can be used to model a large class of practical interference relationships, including IEEE 802.11 DCF [17], [19], Bluetooth, and FH-CDMA [26].

A key parameter that we will frequently use in this paper for characterizing the performance of control algorithms is the interference degree [19], [20]. We first define a non-interfering subset of $I_{l}$ as a subset of links in $I_{l}$ such that any two links in this subset do not interfere with each other. The interference degree $\mathcal{K}(l)$ of link $l$ is the maximum cardinality of any non-interfering subset of $I_{l}$. In other words, the interference degree $\mathcal{K}(l)$ of link $l$ characterizes the potential loss of system capacity if link $l$ is scheduled, i.e., it is the maximum number of links that could have been turned on simultaneously (without interference) if link $l$ was not turned on. The interference degree $\mathcal{K}$ of the whole network is the maximum possible interference degree over all links. Note that the interference degree of the system can often be determined directly from the physical interference model, and thus is independent of the exact network topology. For example, under the so-called node-exclusive interference model for Bluetooch and FH-CDMA networks, the interference degree is 2. For the so-called bi-directional equal-power model that approximates IEEE 802.11 DCF, the interference degree is 8 [19].

Let $M_{i}$ be the number of radio interfaces available at node $i$. We assume that at any given time a radio can only tune to one channel. Therefore, for link $l$ to successfully communicate on channel $c$, both the transmitting node $b(l)$ and the receiving node $e(l)$ must tune one radio to channel $c$. Like [1], [2], [5], [6], [8], [13], we assume that radios can switch channels dynamically.

There are $S$ users in the system. Each user is associated with a source node and a destination node. The traffic from each user may be routed over multiple alternate paths. Let $J(s)$ denote the number of alternate paths for user $s$. Let $\left[H_{s j}^{l}\right]$ denote the routing matrix, where $H_{s j}^{l}=1$ if path $j$ of user $s$ traverses link $l, H_{s j}^{l}=0$, otherwise. For simplicity, we assume that user $s$ injects packets into the system at a constant rate $\lambda_{s}$ (while our results can also be easily generalized to the case 
when packets arrive according to some stationary and ergodic processes).

We assume that time is divided into slots of unit length, and the time that it takes to switch radios between channels is negligible compared to the length of each time slot. For ease of exposition, in the rest of the paper whenever there is no source of confusion, we will use the term "schedule" to refer to both the channel assignment and the link schedule at a time slot. At time slot $t$, let $\mathcal{M}(t)=\left[\mathcal{M}^{c}(t)\right]$ denote the outcome of the scheduling algorithm, where $\mathcal{M}^{c}(t)$ is the set of non-interfering links that are chosen to transmit information at channel $c$ at time $t$. Let $D_{l}(t)$ denote the number of packets that link $l$ can serve at time slot $t$. Then $D_{l}(t)=\sum_{c: l \in \mathcal{M}^{c}(t)} r_{l}^{c}$. Let $P_{s j}(t)$ denote the fraction of traffic from user $s$ that is routed to path $j$ at time slot $t$. Further, let $q_{l}(t)$ denote the number of packets queued at link $l$ at the beginning of time slot $t$. The evolution of $q_{l}(t)$ may be written as:

$$
q_{l}(t+1)=\left[q_{l}(t)+\sum_{s=1}^{S} \sum_{j=1}^{J(s)} H_{s j}^{l} P_{s j}(t) \lambda_{s}-D_{l}(t)\right]^{+},
$$

where $[\cdot]^{+}$denote the projection to $[0,+\infty)$. We say that the system is stable if the queue lengths at all links remain finite [15], i.e.

$$
\lim _{T \rightarrow \infty} \frac{1}{T} \sum_{t=1}^{T} \mathbf{1}_{\left\{\sum_{l=1}^{L} q_{l}(t)>\eta\right\}} \rightarrow 0, \text { almost surely as } \eta \rightarrow \infty .
$$

Remark: Note that in (1) we have adopted the simplifying assumption that packets from each user $s$ are applied to all links $l$ along the path of user $s$ simultaneously. This assumption simplifies the analysis of the paper, and allows us to focus on the channel assignment and scheduling components of the problem. In reality, packets have to traverse the links one at a time. There are a number of known methodologies that can extend our model to take into account this link-by-link packet dynamics [16]-[18], [22]. One such approach is to assume that users can communicate the amount of transmitted traffic $P_{s j}(t) \lambda_{s}$ to all links along path $j$ through an additional control channel, and let each link $l$ update a "virtual" queue according to (1). Then, with an appropriate packet scheduling policy at each link, one can show that the real queue (with link-bylink packet dynamics) is stable as long as the "virtual" queue defined by (1) is stable [16], [27]. Hence, in this paper we will neglect the link-by-link packet dynamics, and use (1) to describe the queue dynamics.

Let $\vec{\lambda}=\left[\lambda_{1}, \ldots \lambda_{S}\right]$ denote the offered load to the network. The capacity region under a particular channel-assignment, scheduling and routing algorithm is the set of $\vec{\lambda}$ such that the system remains stable. Under possible routing constraints, we define the optimal capacity region $\Omega$ as the supremum of the capacity regions of all algorithms. An algorithm is throughputoptimal if it can achieve the optimal capacity region $\Omega$. The efficiency ratio of an algorithm is the largest number $\gamma$ such that this algorithm can stablize the system under any load $\vec{\lambda} \in \gamma \Omega$. By definition, a throughput-optimal algorithm has an efficiency ratio of 1 .

\section{Generalizations of Single-Channel Scheduling Algorithms to Multi-Channel NETWORKS}

In this paper, we are interested in distributed control algorithms with provable efficiency ratios. A number of provablyefficient and low-complexity scheduling algorithms have been proposed for single-channel multi-hop wireless networks [16][22]. One would naturally hope that the generalization of these single-channel scheduling algorithms may lead to equally efficient and low-complexity scheduling algorithms for multichannel networks. In this section, we will study the generalization of two such single-channel scheduling algorithms, i.e., Greedy Maximal Scheduling and Maximal Scheduling, to multi-channel networks. We will first show that the generalization of Greedy Maximal Scheduling, which is a low-complexity but centralized algorithm, can still guarantee efficiency-ratios almost as tight as in single-channel networks. While this result is encouraging, the Greedy Maximal Scheduling algorithm is not very easy to implement in a distributive fashion. It would be more desirable that we can develop distributed scheduling algorithms for multi-channel ad hoc wireless networks. However, we will show that straightforward extensions of Maximal Scheduling, a low-complexity and distributed algorithm, can result in much lower efficiency ratios in multi-channel networks.

\section{A. Greedy Maximal Scheduling}

It is known that the maximum system capacity can be attained by the following throughput-optimal scheduling algorithm [14]-[16]: at each time slot $t$, the schedule $\mathcal{M}(t)$ should be chosen to maximize the queue-weighted rate-sum $\sum_{(l, c): l \in \mathcal{M}^{c}(t)} q_{l}(t) r_{l}^{c}$. The Greedy Maximal Scheduling algorithm can be viewed as an approximation to such throughputoptimal algorithms. Roughly speaking, a schedule $\mathcal{M}$ is maximal if $\mathcal{M}$ is a non-interfering schedule, and no more links can be added to $\mathcal{M}^{c}(t)$ at any channel $c$ without violating the interference constraint and radio interface constraint. The Greedy Maximal Scheduling algorithm computes a maximal schedule by always starting from the link-channel pair with the largest queue-weighted rate $q_{l}(t) r_{l}^{c}$. Specifically, the Greedy Maximal Scheduling algorithm proceeds as follows. (i) Form a set $\mathcal{F}$ of all link-channel pairs $(l, c)$. Define the weight of each link-channel pair $(l, c)$ to be $q_{l} r_{l}^{c}$, where $q_{l}$ is the current queue length at link $l$. Start from an empty schedule $\mathcal{M}(t)$. (ii) First search for the link-channel pair $(l, c)$ with the largest weight $q_{l} r_{l}^{c}$. Add link $l$ to $\mathcal{M}^{c}(t)$. Remove from $\mathcal{F}$ all link-channel pairs that cannot be scheduled due to $(l, c)$ being scheduled. Specifically, remove from $\mathcal{F}$ all link-channel pairs $(k, c)$ with $k \in I_{l}$. (Note that $(l, c)$ is also removed since we define $l \in I_{l}$ ). Further, if by scheduling link $l$ on channel $c$, the transmitting node $b(l)$ (or respectively, the receiving node $e(l)$ ) of link $l$ already uses up all $M_{b(l)}$ (respectively, $\left.M_{e(l)}\right)$ radio interfaces, remove from $\mathcal{F}$ all link-channel pairs $\left(k, c^{\prime}\right)$ 
with $k \in E(b(l))$ (or respectively, $k \in E(e(l))$ ). (In other words, all links incident to node $b(l)$ or $e(l)$ are removed from $\mathcal{F}$ since no radio interfaces are available.) (iii) Next, find the link-channel pair $(l, c)$ with the largest weight $q_{l} r_{l}^{c}$ from the remaining pairs in $\mathcal{F}$, and similar to the previous step, remove from $\mathcal{F}$ all link-channel pairs that cannot be scheduled due to the new $(l, c)$. Continue in this fashion until no link-channel pairs are left in $\mathcal{F}$. Note that the above Greedy Maximal Scheduling algorithm is a natural generalization of the Greedy Maximal Scheduling algorithm for single-channel networks [16] and high-speed switches [28].

The following proposition shows that Greedy Maximal Scheduling can guarantee an efficiency ratio of $1 /(\mathcal{K}+2)$ in multi-channel networks, where $\mathcal{K}$ is the interference degree defined in Section II. Note that the Greedy Maximal Scheduling algorithm has been shown to achieve an efficiency ratio of $1 / \mathcal{K}$ in single-channel networks [16]. Thus, we can conclude that its performance in multi-channel networks is similar. For simplicity, we only present the result for the case where each user has one fixed path through the network. Let $H_{s}^{l}=1$ if the path of user $s$ uses link $l, H_{s}^{l}=0$, otherwise. The proof is provided in our online technical report [29].

Proposition 1: Assume that each user can only use one path, and the routing matrix is given by $\left[H_{s}^{l}\right]$. The above Greedy Maximal Scheduling algorithm can achieve an efficiency ratio of $1 /(\mathcal{K}+2)$. Further, if $M_{i}=C$ for all $i$, i.e., there are no radio interface constraints, Greedy Maximal Scheduling can achieve an efficiency ratio of $1 / \mathcal{K}$.

\section{B. Maximal Scheduling}

The Greedy Maximal Scheduling algorithm is essentially a centralized algorithm, and is not very easy to implement in a distributive fashion. We next turn to extensions of Maximal Scheduling to multi-channel ad hoc wireless networks. Recall the definition of a maximal schedule in Section III-A. Mathematically, a maximal schedule can be stated as follows.

Multi-Channel Maximal Scheduling: $\mathcal{M}(t)$ consists of links $l$ that are backloged, i.e., $q_{l}(t) \geq \sum_{c=1}^{C} r_{l}^{c}$. Further, for any link-channel pair $(l, c)$ such that link $l$ is backloged, at least one of the following is true:

- Either link $l$ is scheduled in channel $c$, i.e., $l \in \mathcal{M}^{c}(t)$, or,

- One of the interfering links to link $l$ is backloged and scheduled in channel $c$, i.e. $k \in \mathcal{M}^{c}(t)$ for some $k \in I_{l}$, or

- Either the transmitter or the receiver of link $l$ has used up all the radios, i.e. $\sum_{k \in E(b(l))} \sum_{d=1}^{C} \mathbf{1}_{\left\{k \in \mathcal{M}^{d}(t)\right\}}=M_{b(l)}$, or $\sum_{k \in E(e(l))} \sum_{d=1}^{C} \mathbf{1}_{\left\{k \in \mathcal{M}^{d}(t)\right\}}=M_{e(l)}$.

Maximal schedules can be easily computed via a distributed algorithm [30]. For single-channel networks, such Maximal Scheduling algorithms have been shown to achieve an efficiency ratio of $1 / \mathcal{K}$. However, for multi-channel networks with channel diversity, such algorithms can perform much worse, because the algorithm could pick the weakest (i.e., least capacity) links at each channel into the maximal schedule. A slightly improved version of Maximal Scheduling for multichannel networks would be to enforce an additional constraint that the scheduling pattern on all channels must be the same. Assume that $M_{i}=C$ for all node $i$, i.e., each node has enough radio interfaces to access all channels simultaneously. Thus, if we aggregate all channels together, the capacity of link $l$ is $\sum_{c=1}^{C} r_{l}^{c}$. We can then define the following Aggregated Maximal Scheduling as in single-channel networks.

\section{Aggregated Maximal Scheduling:}

- If link $l$ is scheduled, it will transmit over all channels simultaneously.

- For any link $l$ that is backloged (i.e., $q_{l} \geq \sum_{c=1}^{C} r_{l}^{c}$ ), either link $l$ is scheduled, or some other backloged link $k \in I_{l}$ is scheduled.

Note that this is in fact the way current IEEE 802.11a standard uses OFDM (readers can refer to the discussions in the Introduction regarding the relationship between OFDM and multi-channel networks). However, the performance of Aggregated Maximal Scheduling can still be very poor. In fact, the following example shows that its efficiency ratio can be as low as $1 / \bar{I}$, where $\bar{I}$ is the maximum number of links that interfere with any link $l$. To see this, consider a node 0 communicating with nodes $1, \ldots, \bar{I}$. Label the link between node 0 and node $i$ as link $i$. Assume that each link interferes with all other links if they operate on the same channel. Hence, only one link can be assigned to each channel at any time. Assume that the total number of channels is also $\bar{I}$. Let the capacity of link $i$ at channel $i$ to be 1 , while its capacity at all other channels is $\epsilon$. Thus, if we use Aggregated Maximal Scheduling, the aggregate capacity of link $i$ is $1+\epsilon(\bar{I}-1)$. Since only one link can be activated at each time, the total capacity of the system is $1+\epsilon(\bar{I}-1)$. However, if we assign each link $i$ to operate on channel $i$, there will be no interference in the system, and the total capacity of the system is thus $\bar{I}$. Clearly, as $\epsilon$ approaches zero, the efficiency ratio of Aggregated Maximal Scheduling can be arbitrarily close to $1 / \bar{I}$. In contrast, Greedy Maximal Scheduling can guarantee the efficiency ratio $1 /(\mathcal{K}+2)$, where $\mathcal{K}$ is independent of network topology.

The above example clearly illustrates the weakness of Maximal Scheduling algorithms in multi-channel networks with channel diversity. In particular, the above extensions of Maximal Scheduling to multi-channel networks fail to take into account the channel diversity, and hence the performance of the scheduling decisions can be very poor.

\section{A Distributed AND PRovably-EFFicient Multi-Channel Control Algorithm}

Given the results in Section III, a natural question is then: can we develop a distributed scheduling algorithm for multi-channel multi-radio ad hoc wireless networks that can guarantee the same efficiency ratio as the centralized Greedy Maximal Scheduling algorithm? In this section, we will develop such a distributed scheduling algorithm. Interestingly, our new scheduling algorithm still uses maximal schedules. Obviously, in order to avoid the inefficiency illustrated in 
Section III-B, we must be able to properly take into account channel diversity. In this work, we introduce a novel twostage queueing mechanism to address channel diversity, and to prevent links from using channels that are weak (i.e., with smaller capacity).

The basic idea of two-stage queueing is as follows. Packets arriving to each link $l$ are served in two steps. The first step is a logical assignment of the packets to channels: packets that arrive at each link $l$ are assigned to queues that correspond to each channel $c$. The second step is the actual scheduling of radio interfaces and links: radios are assigned to channels according to maximal schedules, and packets in the channel queues are served. The key of two-stage queueing is to ensure that, in the first step, packets are less likely to be assigned to link-channel pairs that are "weak." Thus, the "weak" links do not even participate in the maximal schedules in the second step. Clearly, the main difficulty is how to determine in a distributive and online fashion which link-channel pairs are "weak." As we will show next, our algorithm makes this decision intelligently by using the queue length information at both the per-link level and the per-channel level.

\section{A. The Single-Path Case}

For ease of exposition, in this subsection we first focus on the case where each user only has one fixed path through the network. Let $H_{s}^{l}=1$ if the path of user $s$ uses link $l$, $H_{s}^{l}=0$, otherwise. Our proposed multi-channel multi-radio scheduling algorithm works as follows. Each link maintains $C+1$ queues. There is one queue $q_{l}$ for each link $l$, which represents the backlog of packets at link $l$ that have not been assigned to channel queues yet. At the same time, each link $l$ maintains $C$ channel-queues $\eta_{l}^{c}, c=1, \ldots, C$. The per-channel queue $\eta_{l}^{c}$ represents the backlog of packets assigned to channel $c$ by link $l$ that are still waiting to be served. At each time slot $t$, the following algorithm is executed:

\section{Algorithm $S P$ :}

- Step 1: Define $x_{l}^{c}(t)$ to be the number of packets that link $l$ can assign to channel $c$ at time-slot $t$. For each link $l$, let

$$
\begin{aligned}
& x_{l}^{c}(t)=r_{l}^{c}, \text { if } \frac{q_{l}}{\alpha_{l}} \geq \frac{1}{r_{l}^{c}}\left[\sum_{k \in I_{l}} \frac{\eta_{k}^{c}}{r_{k}^{c}}\right. \\
& \left.+\frac{1}{M_{b(l)}} \sum_{k \in E(b(l))} \sum_{d=1}^{C} \frac{\eta_{k}^{d}}{r_{k}^{d}}+\frac{1}{M_{e(l)}} \sum_{k \in E(e(l))} \sum_{d=1}^{C} \frac{\eta_{k}^{d}}{r_{k}^{d}}\right] \\
& x_{l}^{c}(t)=0, \text { otherwise, }
\end{aligned}
$$

where $\alpha_{l}$ is an arbitrary positive constant chosen for link $l$. Then, link $l$ drains $\min \left\{q_{l}(t), \sum_{c=1}^{C} x_{l}^{c}(t)\right\}$ packets from $q_{l}$, and assign them to each channel queue $\eta_{l}^{c}$. Let $y_{l}^{c}(t) \in\left[0, x_{l}^{c}(t)\right]$ be the actual number of packets assigned to each channel queue $\eta_{l}^{c}$. Recall that in the same time slot, link $l$ also receives $\sum_{s=1}^{S} H_{s}^{l} \lambda_{s}$ new packets. The evolution of $q_{l}$ is thus given by:

$$
q_{l}(t+1)=q_{l}(t)+\sum_{s=1}^{S} H_{s}^{l} \lambda_{s}-\sum_{c=1}^{C} y_{l}^{c}(t),
$$

where $\sum_{c=1}^{C} y_{l}^{c}(t)=\min \left\{q_{l}(t), \sum_{c=1}^{C} x_{l}^{c}(t)\right\}$.

- Step 2: Based on the channel queues $\eta_{l}^{c}(t)$, Multi-Channel Maximal Scheduling (as in Section III-B) is carried out to determine the channel-assignment and link schedules. Mathematically, this means that $\mathcal{M}^{c}(t)$ again consists of links $l$ that are backloged in channel $c$ (which is now defined as $\eta_{l}^{c}(t) \geq r_{l}^{c}$ ). Further, for any linkchannel pair $(l, c)$ that is backloged, one of the statements under the Multi-Channel Maximal Scheduling algorithm in Section III-B must hold. Then at the end of step 2, the evolution of each channel queue $\eta_{l}^{c}(t)$ is given by

$$
\eta_{l}^{c}(t+1)=\eta_{l}^{c}(t)+y_{l}^{c}(t)-r_{l}^{c} \mathbf{1}_{\left\{l \in \mathcal{M}^{c}(t)\right\}} .
$$

Remark: The assignment in (2) is the key to ensure that links will only be scheduled on their "strong" channels. Note that in (2) each link only needs to know the channelqueue-length $\eta_{l}^{c}$ at interfering links. This equation can be best explained by interpreting the quantities $q_{l}$ and $\eta_{l}^{c}$ as "price" signals. The quantity $q_{l}$ can be interpreted as the congestion cost at link $l$ (due to the imbalance between the external arrivals and the system capacity). The quantity $\sum_{k \in I_{l}} \frac{\eta_{k}^{c}}{r_{k}^{c}}$ can be interpreted as the contention cost at link $l$ (due to the interference on channel $c$ from links in the interference set $\left.I_{l}\right)$. The quantities $\frac{1}{M_{b(l)}} \sum_{k \in E(b(l))} \sum_{d=1}^{C} \frac{\eta_{k}^{d}}{r_{k}^{d}}$ and $\frac{1}{M_{e(l)}} \sum_{k \in E(e(l))} \sum_{d=1}^{C} \frac{\eta_{k}^{d}}{r_{k}^{d}}$ can be interpreted as the radio costs at the transmitter node $b(l)$ and receiver node $e(l)$, respectively, of link $l$. Hence, each link $l$ will assign traffic to channel $c$ at the maximum rate $r_{l}^{c}$ only if the contention cost of the channel plus the radio cost, weighted by the channel capacity $r_{l}^{c}$, is smaller than the congestion level at the link. Note that if $r_{l}^{c}$ is small, the right hand side of (2) will increase, and hence it is less likely that link $l$ will assign traffic to channel $c$. On the other hand, if the congestion level $q_{l}$ is too high, it is still possible for link $l$ to assign traffic to a channel $c$ with low rate, provided that the other costs are low.

The following main result shows that the efficiency ratio of Algorithm $S P$ is identical to that of the centralized Greedy Maximal Scheduling algorithm of Section III-A.

Proposition 2: Assume that each user can only use one path, and the routing matrix is given by $\left[H_{s}^{l}\right]$. The efficiency ratio of our proposed algorithm $S P$ is $1 /(\mathcal{K}+2)$ where $\mathcal{K}$ is the interference degree defined in Section II.

Proof: We will show that for any $\vec{\lambda}$ such that some scheduling algorithm can stabilize the network at the offered load $(\mathcal{K}+2) \vec{\lambda}$, Algorithm $S P$ will stablize the system at the offered load $\vec{\lambda}$. We use the following Lyapunov function to establish stability:

$$
\begin{aligned}
& V(\vec{q}, \vec{\eta})=\sum_{l=1}^{L} \frac{\left(q_{l}\right)^{2}}{2 \alpha_{l}}+\sum_{l=1}^{L} \sum_{c=1}^{C} \frac{\eta_{l}^{c}}{2 r_{l}^{c}}\left[\sum_{k \in I_{l}} \frac{\eta_{k}^{c}}{r_{l}^{c}}\right. \\
& \left.+\frac{1}{M_{b(l)}} \sum_{k \in E(b(l))} \sum_{d=1}^{C} \frac{\eta_{k}^{d}}{r_{k}^{d}}+\frac{1}{M_{e(l)}} \sum_{k \in E(e(l))} \sum_{d=1}^{C} \frac{\eta_{k}^{d}}{r_{k}^{d}}\right] .
\end{aligned}
$$


Using (3) and (4), the drift $\Delta V(t)=V(\vec{q}(t+1), \vec{\eta}(t+1))-$ $V(\vec{q}(t), \vec{\eta}(t))$ can be bounded by (see [29] for details):

$$
\begin{array}{r}
\mathbf{E}[\Delta V(t) \mid \vec{q}(t), \vec{\eta}(t)] \leq \sum_{l=1}^{L} \frac{q_{l}(t)}{\alpha_{l}}\left[\sum_{s=1}^{S} H_{s}^{l} \lambda_{s}-\sum_{c=1}^{C} x_{l}^{c}(t)\right] \\
+\sum_{l=1}^{L} \sum_{c=1}^{C} \frac{\eta_{l}^{c}(t)}{r_{l}^{c}}\left[\sum_{k \in I_{l}} \frac{x_{k}^{c}(t)}{r_{k}^{c}}+\frac{1}{M_{b(l)}} \sum_{k \in E(b(l))} \sum_{d=1}^{C} \frac{x_{k}^{d}(t)}{r_{k}^{d}}\right. \\
\left.+\frac{1}{M_{e(l)}} \sum_{k \in E(e(l))} \sum_{d=1}^{C} \frac{x_{k}^{d}(t)}{r_{k}^{d}}-\mu_{l}^{c}(t)\right]+C_{4},
\end{array}
$$

where $C_{4}$ is a positive constant and

$$
\begin{aligned}
\mu_{l}^{c}(t)= & \sum_{k \in I_{l}} \mathbf{1}_{\left\{k \in \mathcal{M}^{c}(t)\right\}}+\frac{1}{M_{b(l)}} \sum_{k \in E(b(l))} \sum_{d=1}^{C} \mathbf{1}_{\left\{k \in \mathcal{M}^{d}(t)\right\}} \\
& +\frac{1}{M_{e(l)}} \sum_{k \in E(e(l))} \sum_{d=1}^{C} \mathbf{1}_{\left\{k \in \mathcal{M}^{d}(t)\right\}} .
\end{aligned}
$$

Since there exists some scheduling algorithm that can stabilize the system at the offered load vector $(\mathcal{K}+2) \vec{\lambda}$, there must exist some $\tilde{x}_{l}^{c} \in\left[0, r_{l}^{c}\right]$ for each $(l, c)$ such that

$$
\begin{aligned}
& (1+\epsilon)^{2}(\mathcal{K}+2) \sum_{s=1}^{S} H_{s}^{l} \lambda_{s} \leq \sum_{c=1}^{C} \tilde{x}_{l}^{c}, \text { for all link } l, \\
& \sum_{k \in I_{l}} \frac{\bar{x}_{k}^{c}}{r_{k}^{c}} \leq \mathcal{K}, \text { for all link } l \text { and channel } c \\
& \sum_{k \in E(i)} \sum_{c=1}^{C} \frac{\bar{x}_{k}^{c}}{r_{k}^{c}} \leq M_{i}, \text { for all node } i,
\end{aligned}
$$

where $\tilde{x}_{l}^{c}$ can be interpreted as the long-term average amount of service that link $l$ received at channel $c$, and $\epsilon$ is a small positive number. Note that the inequality (7) is due to the ratebalance at link $l$. The inequality (8) is due to the interference constraint, i.e., there can be no more than $\mathcal{K}$ links activated simultaneously in any interference range $I_{l}$. The inequality (9) is due to the radio interface constraint, i.e., there can be no more than $M_{i}$ link-channel pairs incident to node $i$ that are activated simultaneously (see [7], [8]). Let $\bar{x}_{l}^{c}=\frac{\tilde{x}_{l}^{c}}{(\mathcal{K}+2)(1+\epsilon)}$. We thus have,

$$
\begin{aligned}
& (1+\epsilon) \sum_{s=1}^{S} H_{s}^{l} \lambda_{s} \leq \sum_{c=1}^{C} \bar{x}_{l}^{c}, \text { for all link } l, \\
& (1+\epsilon)\left[\sum_{k \in I_{l}} \frac{\bar{x}_{k}^{c}}{r_{k}^{c}}+\frac{1}{M_{b(l)}} \sum_{k \in E(b(l))} \sum_{d=1}^{C} \frac{\bar{x}_{k}^{d}}{r_{k}^{d}}\right. \\
& \left.+\frac{1}{M_{e(l)}} \sum_{k \in E(e(l))} \sum_{d=1}^{C} \frac{\bar{x}_{k}^{d}}{r_{k}^{d}}\right] \leq 1, \text { for all }(l, c) .
\end{aligned}
$$

Therefore, we have

$$
\begin{aligned}
& \mathbf{E}[\Delta V(t) \mid \vec{q}(t), \vec{\eta}(t)] \\
\leq & \sum_{l=1}^{L} \frac{q_{l}(t)}{\alpha_{l}}\left[\sum_{s=1}^{S} H_{s}^{l} \lambda_{s}-\sum_{c=1}^{C} \bar{x}_{l}^{c}\right]
\end{aligned}
$$

$$
\begin{aligned}
& +\sum_{l=1}^{L} \frac{q_{l}(t)}{\alpha_{l}}\left[\sum_{c=1}^{C} \bar{x}_{l}^{c}-\sum_{c=1}^{C} x_{l}^{c}(t)\right] \\
& +\sum_{l=1}^{L} \sum_{c=1}^{C} \frac{\eta_{l}^{c}(t)}{r_{l}^{c}}\left[\sum_{k \in I_{l}} \frac{\bar{x}_{k}^{c}}{r_{k}^{c}}+\frac{1}{M_{b(l)}} \sum_{k \in E(b(l))} \sum_{d=1}^{C} \frac{\bar{x}_{k}^{d}}{r_{k}^{d}}\right. \\
& \left.+\frac{1}{M_{e(l)}} \sum_{k \in E(e(l))} \sum_{d=1}^{C} \frac{\bar{x}_{k}^{d}}{r_{k}^{d}}-\mu_{l}^{c}(t)\right] \\
& +\sum_{l=1}^{L} \sum_{c=1}^{C} \frac{\eta_{l}^{c}(t)}{r_{l}^{c}}\left[\sum_{k \in I_{l}} \frac{x_{k}^{c}(t)-\bar{x}_{k}^{c}}{r_{k}^{c}}\right. \\
& +\frac{1}{M_{b(l)}} \sum_{k \in E(b(l))} \sum_{d=1}^{C} \frac{x_{k}^{d}(t)-\bar{x}_{k}^{d}}{r_{k}^{d}} \\
& \left.+\frac{1}{M_{e(l)}} \sum_{k \in E(e(l))} \sum_{d=1}^{C} \frac{x_{k}^{d}(t)-\bar{x}_{k}^{d}}{r_{k}^{d}}\right]+C_{4} .
\end{aligned}
$$

Let $m_{l}^{c}=\left[\sum_{k \in I_{l}} \frac{\bar{x}_{k}^{c}}{r_{k}^{c}}+\frac{1}{M_{b(l)}} \sum_{k \in E(b(l))} \sum_{d=1}^{C} \frac{\bar{x}_{k}^{d}}{r_{k}^{d}}+\right.$ $\left.\frac{1}{M_{e(l)}} \sum_{k \in E(e(l))} \sum_{d=1}^{C} \frac{\bar{x}_{k}^{d}}{r_{k}^{d}}\right]$. By definition of Multi-Channel Maximal Scheduling (see Section III-B), $\mu_{l}^{c}(t) \geq 1$ whenever $\eta_{l}^{c}(t) \geq r_{l}^{c}$. Using (10)-(11), we thus have

$$
\begin{gathered}
\mathbf{E}[\Delta V(t) \mid \vec{q}(t), \vec{\eta}(t)] \\
\leq-\epsilon \sum_{l=1}^{L} \frac{\sum_{s=1}^{S} H_{s}^{l} \lambda_{s}}{\alpha_{l}} q_{l}(t)-\epsilon \sum_{l=1}^{L} \sum_{c=1}^{C} \frac{m_{l}^{c}(t)}{r_{l}^{c}} \eta_{l}^{c}(t) \\
+\sum_{l=1}^{L} \sum_{c=1}^{C} \frac{\bar{x}_{l}^{c}-x_{l}^{c}(t)}{r_{l}^{c}}\left[\frac{r_{l}^{c} q_{l}(t)}{\alpha_{l}}-\left(\sum_{k \in I_{l}} \frac{\eta_{k}^{c}(t)}{r_{k}^{c}}\right.\right. \\
+\frac{1}{M_{b(l)}} \sum_{k \in E(b(l))} \sum_{d=1}^{C} \frac{\eta_{k}^{d}(t)}{r_{k}^{d}} \\
\left.\left.+\frac{1}{M_{e(l)}} \sum_{k \in E(e(l))} \sum_{d=1}^{C} \frac{\eta_{k}^{d}(t)}{r_{k}^{d}}\right)\right]+C_{5} .
\end{gathered}
$$

Recall that $0 \leq \bar{x}_{l}^{c} \leq r_{l}^{c}$. Then, by Step 1 of Algorithm $S P$, the third term in (13) is non-positive. The stability of the system then follows [15].

Q.E.D.

If $M_{i}=C$ for all node $i$, i.e., when there are no radio interface constraints, a tighter efficiency ratio can be shown by slightly modifying Algorithm $S P$. In particular, we can remove from (2) the radio costs (i.e., the last two terms). We can then use the following Lyapunov function:

$$
V(\vec{q}, \vec{\eta})=\sum_{l=1}^{L} \frac{\left(q_{l}\right)^{2}}{2 \alpha_{l}}+\sum_{l=1}^{L} \sum_{c=1}^{C} \frac{\eta_{l}^{c}}{2 r_{l}^{c}}\left[\sum_{k \in I_{l}} \frac{\eta_{k}^{c}}{r_{k}^{c}}\right] .
$$

Following the line of proof of Proposition 2, and noting that the last two terms of (11) can also be removed, We can then show the following tighter result.

Proposition 3: Assume that each user can only use one path, and the routing matrix is given by $\left[H_{s}^{l}\right]$. Further, assume 
that $M_{i}=C$ for all node $i$. The efficiency ratio of the modified Algorithm $S P$ is $1 / \mathcal{K}$.

In both cases, the distributed Algorithm $S P$ achieves the same efficiency ratio as the centralized Greedy Maximal Scheduling algorithm in Section III-A.

\section{B. The Multi-Path Case}

We next extend Algorithm $S P$ to the case when each user can use multiple alternate paths. For the moment, we assume that each user is provided with $J(s)$ alternate paths through the network, and we will study how each user should optimally route packets among these alternate paths. Then, in Section IVC, we will discuss how these paths should be computed. Define $P_{s j}$ to be the fraction of incoming packets from user $s$ that are routed to path $j$. Let $\vec{P}_{s}=\left[P_{s 1}, \ldots, P_{s, J(s)}\right]$. Obviously, $P_{s j} \geq 0$ and $\sum_{j=1}^{J(s)} P_{s j}=1$ for all user $s$. Let $\vec{P}=\left[\vec{P}_{1}, \ldots, \vec{P}_{s}\right]$. We can then generalize Algorithm $S P$ to the following joint channel-assignment, scheduling and routing algorithm.

Algorithm $M P$ : At each time slot $t$ :

- Step 1: Each user $s$ computes the routing fractions $P_{s j}(t)$ as the solution to the following local optimization problem:

$$
\begin{aligned}
\max _{\vec{P}_{s}} & -\frac{\beta_{s}}{2} \sum_{j=1}^{J(s)}\left(P_{s j}\right)^{2}-\sum_{j=1}^{J(s)} P_{s j} \sum_{l=1}^{L} H_{s j}^{l} q_{l}(t) \\
\text { subject to } & P_{s j} \geq 0, \sum_{j=1}^{J(s)} P_{s j}=1,
\end{aligned}
$$

where $\left[H_{s j}^{l}\right]$ is the routing matrix, and $\beta_{s}$ is a positive number chosen for each source $s$. Each user $s$ then routes $P_{s j}(t)$ of the arrival traffic to path $j$.

- Step 2: This step is the same as Step 1 of Algorithm $S P$, except that the queue evolution (3) becomes

$$
q_{l}(t+1)=q_{l}(t)+\sum_{s=1}^{S} H_{s j}^{l} P_{s j}(t) \lambda_{s}-\sum_{c=1}^{C} y_{l}^{c}(t) .
$$

- Step 3: This step is the same as Step 2 in Algorithm $S P$. Remark: The local optimization problem (14) is formulated in such a way that the routing fraction $P_{s j}$ is larger for a path $j$ with a smaller congestion cost $\sum_{l=1}^{L} H_{s j}^{l} q_{l}(t)$. Note that each user only needs to know the sum of the queue length $q_{l}$ along its alternate paths. The quadratic term in the objective function of (14) is essential to prevent potential routing oscillation. To see this, note that if $\beta_{s}=0$, then when (14) is solved for any user $s$ that has multiple alternate paths, only paths that have the smallest cost $\sum_{l=1}^{L} H_{s j}^{l} q_{l}(t)$ will have positive $P_{s j}$. This property can easily lead to oscillation of the routing fractions $P_{s j}$ when the queue length $q_{l}$ is being updated [31]. On the other hand, with the addition of a quadratic term, the objective function of (14) becomes strictly concave. The optimal routing fraction then becomes a continuous function of the queue length $q_{l}$.
Thus, routing oscillation is eliminated. The parameter $\beta_{s}$ can also control how sensitive the routing fraction is with respect to the queue length. By setting $\beta_{s}$ to be a larger constant, the routing fraction will become less sensitive to the transient queue dynamics.

The following result shows that Algorithm $M P$ can achieve at least $1 /(\mathcal{K}+2)$ of the maximum system capacity, compared with a throughput-optimal algorithm using the same set of alternate paths. The proof is similar to that of Proposition 2. For details, please refer to [29].

Proposition 4: Assume that the set of alternate paths are given. The efficiency ratio of Algorithm $M P$ is $1 /(\mathcal{K}+2)$. Further, if $M_{i}=C$ for all node $i$, then the efficiency ratio can be improved to $1 / \mathcal{K}$.

Remark: The authors of [7] also developed a joint channel assignment, scheduling and routing algorithm, which has been shown to achieve an efficiency ratio of $\left(\min _{i} M_{i}\right) /(\mathcal{K} C)$ (see Theorem 2 of [7]). The algorithm in [7] is an offline and centralized algorithm. In contrast, Algorithm $M P$ that we developed in this paper is distributed and much simpler. Further, when Algorithm $M P$ uses the same set of paths as computed by the Linear-Programming based algorithm in [7], it can guarantee an efficiency ratio of $1 /(\mathcal{K}+2)$, which is higher than that of [7] if some network nodes only have a small number of radio interfaces. We refer the readers to the discussions in the Introduction regarding the difference between our work and [7], and the potential implications.

\section{How to Generate Alternate Paths}

The set of alternate paths, denoted by the matrix $\left[H_{s j}^{l}\right]$, could potentially be the enumeration of all possible paths between each source-destination pair. In practice, however, a much smaller set of alternate paths suffices. We next describe options to compute and maintain this set of alternate paths. First, we could use the paths computed by any of the routing algorithms in the literature [1], [5], [7]-[11]. Proposition 4 then ensures that the capacity region of Algorithm $M P$ is no worse that $1 /(\mathcal{K}+2)$ times the maximum capacity that can be achieved by using the same set of paths.

A more attractive alternative is to discover paths online. Note that the queue-length $q^{l}$ also provides us with the signal to discover potentially better paths. Given a set of alternate paths, we can easily verify the following property for Step 1 of Algorithm $M P$. For each user $s$, those paths $j$ with positive routing fractions $P_{s j}$ must satisfy the following condition:

$$
\beta_{s} P_{s j}+\sum_{l=1}^{L} H_{s j}^{l} q_{l}=\max _{k=1, . ., J(s)} \beta_{s} P_{s k}+\sum_{l=1}^{L} H_{s k}^{l} q_{l} \triangleq q_{s, \max },
$$

Therefore, adding paths with congestion costs $\sum_{l=1}^{L} H_{s j}^{l} q_{l}$ larger than $q_{s, \max }$ will not yield any gain. We can use this property to iteratively generate the candidate paths online. Starting from any initial set of candidate paths, we execute Algorithm $M P$ for joint routing, channel-assignment and scheduling. Then, from time to time, we can run a minimal cost routing algorithm 


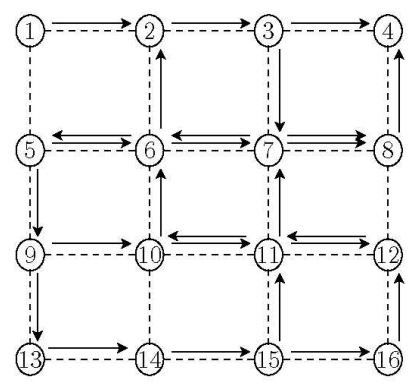

Fig. 1. Network topology

using the queue-length as the cost-metric for each link. If the minimal cost is smaller than $q_{s, \text { max }}$ defined in (15) by a certain threshold, we add this new path into the set of alternate paths, and continue. Otherwise, we can conclude that no further alternate paths need to be added.

\section{Simulation Results}

In this section, we evaluate the performance of our proposed algorithms through simulation. Our simulations are based on the network topology shown in Figure 1. There are 16 nodes (represented by circles) and 24 links (represented by dashed lines). For simplicity, we assume a node-exclusive interference model in our simulations, i.e., only links that share a common node interfere with each other [16], [26]. We also use the simplified equation (1) to model the queue evolution.

We first consider a single-hop scenario. Our goal is to compare the performance of the fixed-path Algorithm $S P$ with Greedy Maximal Scheduling and Aggregated Maximal Scheduling in Section III. We assume that there are 8 available channels in the system, and each node has 8 radios so that it can transmit/receive on 8 channels simultaneously. In our simulation, the capacity $r_{l}^{c}$ of each channel for each link is randomly chosen from 1 to 5 , which models channeldiversity. The single-hop flows are represented by arrows in Fig. 1. We let the rate of each flow be $\lambda$. Note that although the rates of the flows are the same, the composition of the flows has been chosen to avoid uniform patterns. We have chosen the parameter $\alpha_{s}$ in (2) to be 100 for all flows, so that the per-link queues and the channel-queues are on the same order of magnitude. In Figure 2, we plot the peruser average queue backlog, as the packet arrival rate $\lambda$ increases. When $\lambda$ approaches a certain limit, the average queue backlog will increase to infinity. This limit can then be viewed as the boundary of the capacity region. As shown in Fig. 2, the capacity of Algorithm SP is approximately $60 \%$ higher than that of Aggregated Maximal Scheduling. Thus, by exploiting channel-diversity, Algorithm $S P$ can significantly improve the system capacity. Greedy Maximal Scheduling can further improve the capacity by another $30 \%$. However, it requires centralized control and is not easy to implement in a distributive fashion.

Our second set of simulations consider the same network topology in Fig. 1, but now with three available channels

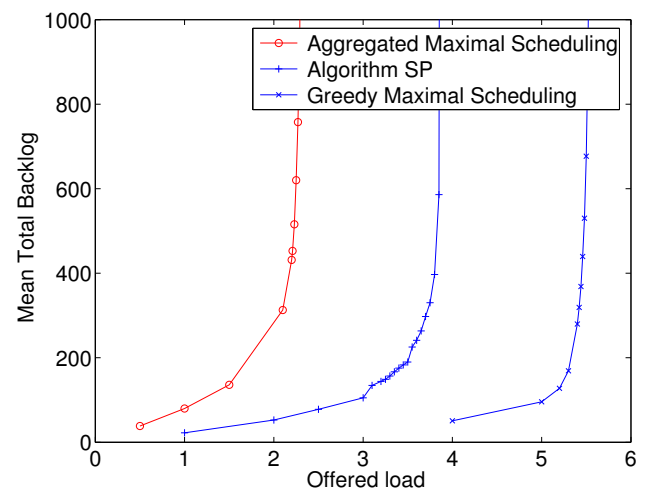

Fig. 2. Comparison of Algorithm $S P$ with Greedy Maximal Scheduling and Aggregated Maximal Scheduling

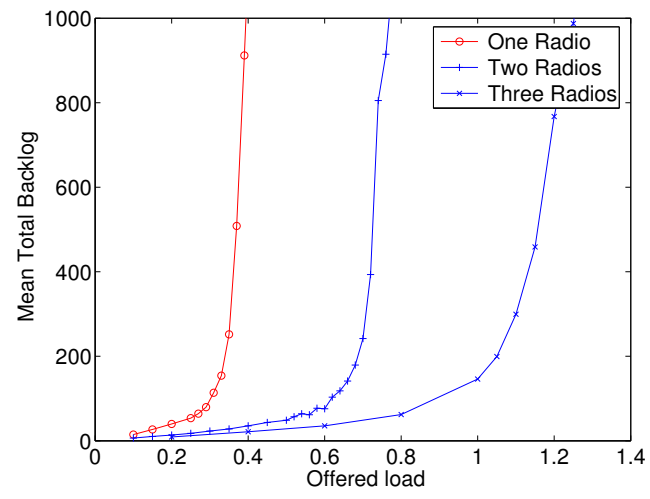

Fig. 3. Performance of Algorithm $M P$.

and with multi-hop communications and multi-path routing. We randomly pick eight source-destination pairs. We then run Algorithm $M P$ with one, two and three radios for each node. In order to limit the complexity of the simulation, we maintain at most four alternate paths for each source-destination pair. Every 200 time-slots, we update the alternate paths according to the online strategy described in Section IV-C. Specifically, we first compute the minimum-cost path using the queue length as the cost metric. Then, the new minimum-cost path is added into the set of alternate paths if its cost is less than $3 / 4$ of the right-hand-side of (15). If there are already four alternate paths between a source-destination pair, the existing path with the smallest routing fraction is replaced by the new path. We also choose the parameter $\beta_{s}$ in (14) to be 0.001 , so that the routing fractions are not too sensitive to the transient queue updates. In Figure 3, we plot the mean network backlog when there are one, two, and three radios for each node. As expected, the network capacity increases as the number of radios increases.

\section{CONCLUSION}

In this paper, we develop a fully distributed algorithm that jointly solve the channel-assignment, scheduling and routing problem for multi-channel multi-radio ad hoc wireless networks. Our algorithm is very simple, does not require prior information on the offered load to the network, and can thus 
adapt automatically to the changes in the network topology and offered load. We show that our algorithm is provably efficient. That is, even compared with the optimal centralized and offline algorithm, our proposed algorithm can achieve a provable fraction of the maximum system capacity. Further, the achievable fraction that we can guarantee is larger than that of the centralized and offline algorithm in [7].

The simplicity of our proposed algorithm and its stronger performance guarantees do motivate many interesting questions as to how the algorithm should be implemented in real networks. We note that, throughout this paper, we have neglected important protocol overheads in our analyses and simulations. In real implementations, additional protocols need to be carefully designed in order to: (i) exchange channelqueue-length information between interfering links so that each link can assign traffic to channel queues according to (2); (ii) compute maximal schedules and inform the transmitter/receiver nodes about which channel(s) they should tune to; (iii) feedback the queue-length information to the source so that the source can make routing decisions according to (14); and (iv) account for actual link-by-link packet dynamics (see the remarks under Equation (1)). Past studies in the literature have suggested that these protocol overheads could be substantially reduced without significantly affecting the actual performance of the algorithm [17], [18], [21], [32]. For future work, we plan to carefully address these protocol issues and study their impact on the actual system performance.

\section{REFERENCES}

[1] P. Kyasanur and N. H. Vaidya, "Routing and Link-layer Protocols for Multi-Channel Multi-Interface Ad Hoc Wireless Networks," SIGMOBILE Mobile Computing and Communications Review, vol. 10, no. 1, pp. 31-43, January 2006.

[2] P. Kyasanur, J. So, C. Chereddi, and N. H. Vaidya, "Multi-Channel Mesh Networks: Challenges and Protocols," IEEE Wireless Communications, April 2006.

[3] C. Chereddi, P. Kyasanur, and N. H. Vaidya, "Design and Implementation of a Multi-Channel Multi-Interface Network," in REALMAN, May 2006.

[4] P. Kyasanur and N. H. Vaidya, "Capacity of Multi-Channel Wireless Networks: Impact of Number of Channels and Interfaces," in ACM Mobicom, Cologne, Germany, August 2005.

[5] J. So and N. H. Vaidya, "Routing and Channel Assignment in MultiChannel Multi-Hop Wireless Networks with Single Network Interface," in 2nd International Conference on Quality of Service in Heterogeneous Wired/Wireless Networks (QShine), August 2005.

[6] — - "Multi-Channel MAC for Ad Hoc Networks: Handling MultiChannel Hidden Terminals Using A Single Transceiver," in ACM International Symposium on Mobile Ad Hoc Networking and Computing (MobiHoc), May 2004.

[7] M. Alicherry, R. Bhatia, and L. Li, "Joint Channel Assignment and Routing for Throughput Optimization in Multi-radio Wireless Mesh Networks," in ACM MobiCom, Cologne, Germany, August 2005.

[8] M. Kodialam and T. Nandagopal, "Characterizing the Capacity Region in Multi-Radio Multi-Channel Wireless Mesh Networks," in ACM MobiCom, Cologne, Germany, August 2005.

[9] A. Raniwala and T. Chiueh, "Architecture and Algorithms for an IEEE 802.11-Based Multi-Channel Wireless Mesh Network," in Proceedings of IEEE INFOCOM, Miami, FL, March 2005.

[10] A. Raniwala, K. Gopalan, and T. Chiueh, "Centralized Algorithms for Multi-channel Wireless Mesh Networks," in ACM Mobile Computing and Communications Review, April 2004.

[11] R. Draves, J. Padhye, and B. Zill, "Routing in Multi-Radio, Multi-Hop Wireless Mesh Networks," in ACM MobiCom, Philadelphia, Pennsylvania, September 2004.
[12] A. Adya, P. Bahl, J. Padhye, A. Wolman, and L. Zhou, "A MultiRadio Unification Protocol for IEEE 802.11 Wireless Networks," in Proceedings of IEEE Broadnets 2004, San Jose, CA, October 2004.

[13] P. Bahl, R. Chandra, and J. Dunagan, "SSCH: Slotted Seeded Channel Hopping for Capacity Improvement in IEEE 802.11 Ad-Hoc Wireless Networks," in Proceedings of ACM MobiCom, Philadelphia, PA, September 2004.

[14] L. Tassiulas and A. Ephremides, "Stability Properties of Constrained Queueing Systems and Scheduling Policies for Maximum Throughput in Multihop Radio Networks," IEEE Transactions on Automatic Control, vol. 37, no. 12, pp. 1936-1948, December 1992.

[15] M. J. Neely, E. Modiano, and C. E. Rohrs, "Dynamic Power Allocation and Routing for Time Varying Wireless Networks," in Proceedings of IEEE INFOCOM, San Francisco, April 2003.

[16] X. Lin and N. B. Shroff, "The Impact of Imperfect Scheduling on CrossLayer Rate Control in Multihop Wireless Networks," in Proceedings of IEEE INFOCOM, Miami, FL, March 2005.

[17] X. Wu and R. Srikant, "Bounds on the Capacity Region of Multihop Wireless Networks Under Distributed Greedy Scheduling," in Proceedings of IEEE INFOCOM, Barcelona, Spain, April 2006.

[18] X. Wu, R. Srikant, and J. R. Perkins, "Queue-Length Stability of Maximal Greedy Schedules in Wireless Network," in Proceedings of Information Theory and Applications Inaugural Workshop, University of California, San Diego, February 2006.

[19] P. Chaporkar, K. Kar, and S. Sarkar, "Throughput Guarantees in Maximal Scheduling in Wireless Networks," in Proceedings of 43rd Annual Allerton Conference on Communication, Control and Computing, Monticello, IL, September 2005.

[20] _ _ "Achieving Queue Length Stability Through Maximal Scheduling in Wireless Networks," in Proceedings of Information Theory and Applications Inaugural Workshop, University of California, San Diego, February 2006.

[21] X. Lin and S. Rasool, "Constant-Time Distributed Scheduling Policies for Ad Hoc Wireless Networks," in Proceedings of the IEEE Conference on Decision and Control, San Diego, CA, December 2006.

[22] X. Wu and R. Srikant, "Regulated Maximal Matching: A Distributed Scheduling Algorithm for Multi-Hop Wireless Networks With NodeExclusive Spectrum Sharing," in Proceedings of IEEE CDC, Seville, Spain, December 2005.

[23] Y. Liu and E. Knightly, "Opportunistic Fair Scheduling over Multiple Wireless Channels," in Proceedings of IEEE INFOCOM, San Francisco, CA, April 2003.

[24] P. Bender, P. Black, M. Grob, R. Padovani, N. Sindhushayana, and A. Viterbi, "CDMA/HDR: a bandwidth-efficient high-speed wireless data service for nomadic users," IEEE Communications magazine, pp. 70-77, July 2000.

[25] X. Liu, E. K. P. Chong, and N. B. Shroff, "A Framework for Opportunistic Scheduling in Wireless Networks," Computer Networks, vol. 41, no. 4, pp. 451-474, March 2003.

[26] B. Hajek and G. Sasaki, "Link Scheduling in Polynomial Time," IEEE Transactions on Information Theory, vol. 34, no. 5, pp. 910-917, September 1988.

[27] S. H. Lu and P. R. Kumar, "Distributed Scheduling Based on Due Dates and Buffer Priorities," IEEE Transactions on Automatic Control, pp. 1406-1416, December 1991.

[28] E. Leonardi, M. Mellia, F. Neri, and M. A. Marsan, "On the Stability of Input-Queued Switches with Speed-Up," IEEE/ACM Transactions on Networking, vol. 9, no. 1, pp. 104-118, February 2001.

[29] X. Lin and S. Rasool, "A Distributed and Provably-Efficient Joint Channel-Assignment, Scheduling and Routing Algorithm for MultiChannel Multi-Radio Wireless Mesh Networks," Technical Report, Purdue University, http://min.ecn.purdue.edu/ linx/papers.html, 2006.

[30] M. Hanckowiak, M. Karonski, and A. Panconesi, "On the Distributed Complexity of Computing Maximal Matching," SIAM Journal of Discrete Mathematics, vol. 15, no. 1, pp. 41-57, 2001.

[31] X. Lin and N. B. Shroff, "Utility Maximization for Communication Networks with Multi-path Routing," IEEE Transactions on Automatic Control, vol. 51, no. 5, pp. 766-781, May 2006.

[32] L. Bui, A. Eryilmaz, R. Srikant, and X. Wu, "Joint Asynchronous Congestion Control and Distributed Scheduling for Multihop Wireless Networks," in Proceedings of IEEE INFOCOM, Barcelona, Spain, April 2006. 